\title{
Retrospective study of Diffuse Intrinsic Pontine Glioma in the Belgian population: a 25 year experience
}

\section{Dries Ruttens ( $\nabla$ dries.ruttens@student.kuleuven.be)}

UZ Leuven: Katholieke Universiteit Leuven Universitaire Ziekenhuizen Leuven https://orcid.org/00000002-1048-1800

\section{Julie Messiaen}

University Hospitals Leuven: Katholieke Universiteit Leuven Universitaire Ziekenhuizen Leuven

\section{Alina Ferster}

HUDERF: Hopital Universitaire des Enfants Reine Fabiola

\section{Caroline Piette}

CHR Citadelle: Centre Hospitalier Regional de la Citadelle

\section{Stefan Schifflers}

Liege University: Universite de Liege

\section{An Van Damme}

University Hospital Saint-Luc: Cliniques Universitaires Saint-Luc

\section{Jutte van der Werff ten Bosch}

UZ Brussel: Universitair Ziekenhuis Brussel

\section{Joris Verlooy}

UZA: Universitair Ziekenhuis Antwerpen

\section{Leen Willems}

UZ Gent: Universitair Ziekenhuis Gent

\section{Sandra Jacobs}

UZ Leuven: Katholieke Universiteit Leuven Universitaire Ziekenhuizen Leuven

\section{Research Article}

Keywords: Diffuse intrinsic pontine glioma, brainstem glioma, diffuse midline glioma, biopsy, radiotherapy, chemotherapy

Posted Date: March 9th, 2021

DOI: https://doi.org/10.21203/rs.3.rs-290956/v1 
License: (c) (i) This work is licensed under a Creative Commons Attribution 4.0 International License. Read Full License 


\section{Abstract \\ Introduction}

Diffuse intrinsic pontine glioma is a rare disease with a high mortality. Our primary aim was to determine the incidence of this disease in Belgium. Secondly, we wanted to compare the treatment approach of Belgian pediatric oncology centres, to investigate possibilities for improvement.

\section{Methods}

We retrospectively collected and analysed data on DIPG-patients diagnosed between 1994 and 2018 and recorded in the Belgian Cancer Registry.

We included patients $\leq 18$ years who were followed in one of the eight Belgian pediatric oncology centres.

\section{Results}

We included 100 patients over a period of 25 years with a median age at diagnosis of 7 years. We observed an increase in diagnoses with an incidence of 3.1 per $1,000,000$ persons (aged $0-\leq 18$ ) per year over the last 5 years compared to an overall incidence of 1.8. Forty-five patients (51.7\%) were biopsied at diagnosis. In ten (22\%), this was study-related. H3 K27M-mutation was present in $75 \%$ of biopsied patients. Fifty-one patients (59.3\%) received chemotherapy, without a significant survival benefit. Eleven patients (21.2\%) were included in a clinical trial. Biopsy rate and the use of chemotherapy differed widely between centres. Mean OS and PFS were 10.49 and 4.87 months respectively. We observed an improved survival over time.

\section{Conclusions}

Over the past 25 years, we observed an increase of new DIPG-diagnoses. Outcome in our cohort is comparable with literature findings. We demonstrate an important heterogeneity in treatment approach between different centres and limited inclusion in clinical trials. Therefore, collaboration between centres and inclusion of patients in clinical trials is much needed.

\section{Introduction}

Diffuse intrinsic pontine glioma (DIPG) is a rare pediatric brain stem malignancy with an unfavourable prognosis. Because of its location, surgical resection is not feasible. To date, there is no treatment able to cure this disease. $(1,2)$ The yearly incidence is 2.32 per $1,000,000$ inhabitants aged $0-20$ years, with a median age at diagnosis of approximately 7 years. The median overall survival (OS) ranges from 8 to 11 
months with an OS of less than $10 \%$ at 2 years. Male and female are equally affected.(3-5) Onset of the disease mostly is acute and common symptoms include cerebellar signs, pyramidal signs and cranial nerve palsy (especially abducens nerve palsy). $(5,6)$ Currently diagnosis mainly relies on magnetic resonance imaging (MRI).(7) The lack of tissue for diagnosis impeded research on the underlying molecular pathogenesis.(6) As a result, the treatment for DIPG was largely based on the treatment of adult high-grade gliomas. With the emergence of surgical biopsy as a safe procedure with acceptable risks, $(8,9)$ our knowledge about the molecular roots of this disease has increased and DIPG has been individualized as a distinct entity. $(10,11)$ An important discovery is the presence of the K27M driving mutation in histone $\mathrm{H} 3$ genes H3F3A, HIST2H3C and HIST1H3B/C, present in almost $80 \%$ of all DIPGs.(4, 11-13) This led to reclassification of most DIPGs as diffuse midline glioma H3 K27M-mutant in the last World Health Organization classification of the central nervous system tumours. This entity encompasses a phenotypically and molecularly defined set of tumours which share their diffuse growth pattern and location in the brain midline, including thalamus, brain stem and spinal cord.(14) Currently, about $42 \%$ of the medical practitioners state to biopsy all or most of their patients,(15) mainly in case of atypical imaging or in the setting of a clinical trial.(16) Focal radiotherapy is the only treatment with a proven survival benefit and remains the standard of care. Conventional fractionation (54-59.4 Gy in

$1.8 \mathrm{~Gy}$ fractions) increases OS with approximately 3 months. Hypofractionation schemes (39 Gy in $3 \mathrm{~Gy}$ fractions) can offer comparable results with a smaller treatment burden. A hyperfractionation (6678 Gy in 1-1.26 Gy fractions) scheme does not improve survival but is more inconvenient and has a higher risk of acute toxicity. $(6,15,17)$ In selected patients, reirradiation at progression can provide symptom relief and a modest survival benefit with acceptable toxicity. $(18,19)$ Numerous chemotherapeutic agents have been tested in multiple clinical trials, although none of these could improve survival.(6) In Belgium, all oncologic patients are registered in the Belgian Cancer Registry. DIPG however, is not registered as a separate entity and no data on incidence, epidemiology, treatment and prognosis are available.

We retrospectively collected data on all Belgian DIPG-patients diagnosed over the past 25 years. Our primary aim was to determine the incidence of this specific entity in Belgium, along with other epidemiological and clinical data. Secondly, we intended to gather information on the treatment approach used in Belgian oncology centres. Comparing our current practice to recent evidence, we aimed to find possibilities to improve our approach.

\section{Material And Methods}

\section{Patients}

We retrospectively collected data on patients diagnosed with DIPG between 1994 and 2018, with an age at diagnosis between 0 and 18 years (age 18 included) who were followed in one of the recognised Belgian pediatric oncology centres. Patients were retrieved from local registries by means of following search terms: 'DIPG' and 'pontine glioma'. Inclusion in each centre was performed analogously, with minor variations according to local data registry. Patients were included in the study if the MRI reports 
were conclusive of a DIPG. Patients were excluded if they had a pathological diagnosis of a pilocytic astrocytoma (WHO grade 1). Patients who were followed in two or more centres were only included once. Data collection was conducted in compliance with the declaration of Helsinki. Approval by The Ethical Committee of all participating sites was acquired before data collection. All data were anonymized and access to the data was restricted to individuals directly linked to the study.

Since it concerned retrospective data collection, informed consent was not required. All data were treated confidentially by the investigator and all participating sites.

\section{Data collection and analysis}

Information was collected on epidemiological and clinical characteristics (medical history and physical examination at time of diagnosis and during follow-up), tumour- and treatment-related data, as well as outcome parameters. We visited all eight recognized Belgian pediatric oncology centres (i.e. CHC

Clinique de l'Espérance, CHU Liège, HUDERF, Cliniques Universitaires Saint-Luc, UZ Antwerpen, UZ Brussel, UZ Gent, UZ Leuven) to retrospectively collect data. In UZ Brussel, data were only available from 2000 onwards. All data were coded and transferred to a database with a patient specific number. If specific data were lacking, the patient was omitted for the regarding analysis. Incidence and survival analyses were performed on the total cohort, whereas all other analyses were performed on 87 patients (unless otherwise stated) due to incompleteness of specific files.

\section{Statistical methods}

The collected data were analysed using IBM SPSS Statistics (version 26) and SAS software (Version 9.4). We mainly applied descriptive statistics. A linear regression analysis was used to estimate evolution of incidence over time. A logistic regression model was used with biopsy as a binary variable and the year of diagnosis as a covariate to evaluate biopsy rate over time. Survival analyses were performed using the Kaplan-Meier method. The evolution of survival over time was analysed using a Cox model with year of diagnosis as a continuous predictor variable. A Cox proportional hazards model was used to compare OS and PFS in different treatment groups. A multivariate analysis was used to correct for possible confounders. All tests were performed using a $5 \%$ two-sided significance level. No correction for multiplicity was performed given the exploratory nature of the study

\section{Results}

\section{Demographics}

In total, 100 patients were included over a period of 25 years. The median age at diagnosis was 7 years (range 1-16), the mean age at diagnosis was 7.7 years (standard deviation 3.5). The male/female ratio was 0.82 . We calculated an incidence of 1.8 per $1,000,000$ persons (aged $0-\leq 18$ ) per year. The yearly incidence calculated over the last 10 and 5 years (respectively from 2009 and 2014 up to and including 2018) were 2.7 and 3.1 respectively. Figure 1 shows new diagnosed cases over time. A linear regression 
analysis was used to estimate evolution of new diagnosed cases per year over time. Two separate estimates were made: one including all years and excluding patients from the centre with data only available from 2000 onwards, another including all patients from 2000 onwards. Both estimates show a statistically significant increase of diagnosed cases over time (slope $10.210(95 \% \mathrm{Cl} 0.132 ; 0.288, \mathrm{p}<$

0.0001), slope 20.314 (95\% Cl 0.125; 0.504, p = 0.0029).

\section{Patient characteristics}

The mean time between onset of symptoms and diagnosis was approximately 8 weeks with $83.8 \%(\mathrm{n}=$ 67 , time of onset of symptoms known for $n=80$ ) of patients presenting less than 12 weeks after onset of symptoms. Cranial nerve deficit, cerebellar signs and long tract signs were present in 80.5, 78.2 and $67.8 \%$ of patients respectively. An abducens or facial nerve palsy were present in $50.6 \%$ and $48.3 \%$ of patients respectively. Symptoms consistent with raised intracranial pressure (i.e. headache, vomiting and/or papilledema) were present in $54 \%$ of the patients compared to hydrocephalus being present in $29.9 \%$ of the patients. Results of histopathological examination of cerebrospinal fluid (CSF) was documented in 7 patients (all of them underwent CSF drainage for hydrocephalus). Tumour cells were present in the CSF of one patient. No spinal metastases were detected in patients that underwent a spinal MRI $(n=24)$. A biopsy was performed at diagnosis in $51.7 \%(n=45)$ of patients. Depending on the centre, a biopsy was performed in $32-83.3 \%$ of patients. The reason to perform a biopsy was confirmation of the diagnosis in $77.8 \%(n=35)$ of patients, compared to $22.2 \%(n=10)$ where this was done as part of a clinical trial. A logistic regression model was used to evaluate biopsy rate over time. There is no evidence for a decrease or increase in biopsy rate (odds ratio $0.994,95 \% \mathrm{Cl} 0.939 ; 1.052, \mathrm{p}=0.8372$ ). Histological information was retrievable for 34 of 45 biopsies. All tumours were WHO-grade 2 or higher with no predominance of either grade. A mutation analysis was documented in 12 patients. There were five cases of H3.3K27M mutation, one case of HIST1H3B mutation and one case of ACVR1 mutation. One patient showed three mutations, namely in the PIK3CA-, PTEN- and ASXL1-gene. In four patients results were not available.

\section{Treatment characteristics}

Treatment data are summarised in Table 1. Of all patients, $81.6 \%(n=71)$ received radiotherapy. In $77.5 \%$ $(n=55)$ a conventional schedule was used, while a hypofractionation schedule was used in $19.7 \%(n=$ 14). Chemotherapy (cytotoxic or targeted therapy) was given to $59.3 \%(n=51)$ of patients. Only $12.6 \%(n$ $=11$ ) got included in a clinical trial. The first reported clinical trial in Europe dates from 2010. From patients diagnosed in 2010 or later, $21.2 \%$ got included in a clinical trial. Of the patients who underwent radiotherapy, 7 patients (9.9\%) were reirradiated at progression. In total 17 different chemotherapeutic agents and regimens were used (Table 2). Temozolomide and the mTOR-inhibitors

Everolimus and Sirolimus were the agents used most frequently, in 21 and 15 patients respectively. When comparing biopsy rate and the use of chemotherapy between the different centres, we found a broad range, even after omitting the centres with only a few patients. The use of chemotherapy ranged from 42.1 to $75 \%$. 


\section{Survival analyses}

At last follow-up, 91 patients had died. For one patient, survival could not be calculated due to lacking date of diagnosis. Progression was recorded in 69 patients. Of the remaining 22 patients, PFS could not be calculated either because the patient showed primary progression or because date of diagnosis or date of progression were not retrievable. Figure 2 shows the Kaplan-Meier plots of the estimated OS and PFS rate of study patients. The median OS was 10.49 months $(95 \% \mathrm{Cl} 9.1 ; 11.7)$. OS rates at 1 and 2 years were $36.9 \%(95 \% \mathrm{Cl} 27.4 ; 46.5)$ and $4.3 \%(95 \% \mathrm{Cl} 1.2 ; 10.5)$ respectively. The median PFS was 4.87 months $(95 \% \mathrm{Cl} 4.1 ; 6.0)$. A Cox model was used to analyse the evolution of survival over time, with year of diagnosis as a continuous predictor variable. The hazard ratio (HR) for a one-year increase was estimated. This showed a HR of 0.951 (95\% $\mathrm{Cl} 0.924 ; 0.978, \mathrm{p}$ 0.0006), corresponding to an improved survival over time. Survival rates in different treatment groups were compared. Figure 3a shows OS rates of patients treated with radiotherapy alone versus treatment with a combination of radiotherapy and chemotherapy. Survival rates in the group that received both treatments are higher at most time points, but the difference in OS between the two treatment groups was not statistically significant (HR $1.635, \mathrm{Cl}$ $0.955 ; 2.799, \mathrm{p} 0.0729)$. We also compared PFS between both treatment groups, but likewise no statistically significant difference could be shown (HR 1.26, Cl 0.697; 2.28, p 0.443). Figure 3b shows OS rates of patients treated with radiotherapy alone versus treatment with a combination of radiotherapy and cytotoxic or targeted therapy or both. There was a better OS in the group treated with radiotherapy and both cytotoxic and targeted therapy (HR 3.106, $\mathrm{Cl} 1.039 ; 9.281, \mathrm{p} \mathrm{0.0424)}$. When comparing the groups treated with radiotherapy and a combination of radiotherapy and targeted therapy, there is a trend towards better OS, although this difference was not statistically significant (HR 1.951, Cl 0.968; 3.932, p 0.0617). We also observed an improved OS in the group treated with radiotherapy and targeted therapy as compared to the group treated with radiotherapy and cytotoxic therapy, but again this was not statistically significant (HR 1.672, $\mathrm{Cl} 0.865 ; 3.232, \mathrm{p} 0.1265)$. There was no statistically significant difference on PFS between treatment groups. Because most of the patients treated with targeted therapy received mTOR-inhibitors, we compared OS between patients who were treated with mTOR-inhibitors and those treated with other targeted therapies. No difference in OS could be detected (HR 1.108, Cl 0.386; $3.182, \mathrm{p}$ 0.8485). To investigate the effect of age at diagnosis on OS, we applied a Cox proportional hazards model to compare OS rates of patients aged less than 5 years or 5 years and more at diagnosis. No statistical difference could be demonstrated (HR

$0.749,95 \%$ Cl $0.465 ; 1.208, \mathrm{p} 0.2363)$.

\section{Discussion}

DIPG is an aggressive disease associated with high morbidity and mortality for which no curative treatment exists. Since this disease is not registered as a separate entity within the Belgian Cancer Registry, we aimed at broadening our knowledge regarding DIPG, by gathering data on all known Belgian DIPG patients over the past 25 years. 
The demographics of our study population are in line with those described in literature. Interestingly, we find a statistically significant increase in the number of new diagnosed cases per year. This can reflect a true increase in incidence, possibly relating to environmental contributing factors. On the other hand, this increase can also reflect previous underreporting. In both cases, this could mean that the actual incidence of DIPG is higher than estimated previously. When we calculate the incidence over the last ten and five years for example, we find an incidence of 2.7 and 3.1 per 1,000,000 persons (aged $0-\leq 18$ ) per year respectively. Assuming that all DIPG cases between 2009 and 2018 were correctly registered, the difference between these incidences cannot be attributed to underreporting. These incidences are somewhat higher than the incidence reported in the Netherlands (i.e. 2.32 per 1,000,000 persons aged 020 per year).(3)

Most patients presented with cerebellar signs and/or a cranial nerve deficit. In addition to the frequent presence of an abducens nerve palsy at diagnosis, $(6,20)$ more than half of the patients presented with a facial nerve palsy. Tumour cells were present in the CSF of one patient. Because there were only few cases of documented histopathology examination of CSF, the importance of this finding is unclear. Considering the finding that $\mathrm{H} 3$ mutations can be detected through analysis of circulating tumour DNA in CSF of patients with a diffuse midline glioma, our finding could further advocate for the use of liquid biopsies to diagnose and monitor this disease. $(21,22)$ This could offer the possibility to replace invasive biopsy, in selected patients. About half of the patients were biopsied at time of diagnosis. The main reason for biopsy was to confirm diagnosis, which is surprising since the mainstay of diagnosis remains MRI findings.(7) We believe that biopsy should not be a routine part of the diagnostic work-up and that biopsy should be reserved for patients who can be included in clinical trials, or for those cases where biopsy results would influence therapeutical decisions. We could not objectivate a change in biopsy rate over time, despite the fact that during the past decade, several studies supported the safety of biopsy in DIPG patients. $(8,9)$ In contrast to this high biopsy rate, a mutation analysis was only performed in 12 patients $(26.7 \%)$. Results of 8 analyses were available and of these, $75 \%(n=6)$ had the H3 K27M mutation. This finding is in keeping with literature findings.(6)

Only a minority of patients was included in clinical trials, a result comparable to a similar study performed in the Netherlands.(3) Given the urgent need for more effective treatments, we underscore the importance of inclusion in prospective clinical trials. The majority of patients received radiotherapy. The main reason for not receiving radiotherapy was advanced disease at diagnosis. Only a minority of patients who received radiotherapy were treated with a hypo-fractionation schedule. However, research demonstrated that hypo-fractionation schedules offer comparable survival.(13) Since hypo-fractionation schedules are associated with a smaller treatment burden, the choice for the latter could positively impact on quality of life. Strikingly, nearly $70 \%$ of patients who started treatment (radiotherapy and/or chemotherapy), received chemotherapy. This is surprising, since there is no proven survival benefit for chemotherapy.(6) Therefore, we think that chemotherapy should only be used in the setting of a clinical trial. When comparing practices between centres, we observe a broad range in biopsy rate and the use of chemotherapy (including the type of agent used). This diversity reveals a lack of uniform treatment guidelines and underscores the need for a common treatment approach. 
OS and PFS in our cohort match literature findings. There were no long-term survivors in our cohort. We observed a statistically significant improvement in OS over time. A possible explanation for this could be the introduction of reirradiation, with 6 of the 7 patients that underwent reirradiation being diagnosed from 2016 onwards. No statistical difference in OS could be demonstrated between patients treated with radiotherapy and chemotherapy compared to radiotherapy alone. This may be related to a true lack of response to chemotherapy or to the fact that all types of chemotherapy were considered collectively. The most likely explanation for the observed improvement in OS of patients treated with radiotherapy and both cytotoxic and targeted therapy, is the fact that patients with a longer survival have a higher chance of receiving more than one line of treatment. In our study population, there was no prognostic significance of age at diagnosis or symptom duration before diagnosis. This is in contrast with literature findings. $(23,24)$

Because of comparable survival results and less toxicity, we think that targeted treatment is preferred over cytotoxic therapy. Combination of radiotherapy and targeted therapy in the setting of a clinical trial most likely is the way forward in search for a better treatment for DIPG. Biopsy could be helpful in this setting to guide treatment.

Limitations of this study are the lack of central imaging review and the fact that not all files were complete, inherent to the retrospective design. Another limitation is the fact that we could not correct for the general condition of the patient at diagnosis when performing survival analyses.

In conclusion, we were able to gather data on 100 DIPG-patients diagnosed in Belgium over the past 25 years. Since patients with DIPG were not separately registered at the Belgian Cancer Registry, this provides important new information. We determined an incidence of DIPG in Belgium of 1.8 per 1,000,000 persons (aged $0-\leq 18$ ) with a statistically significant increase in incidence over time. OS and PFS in our cohort are comparable with the literature. We observed an important heterogeneity in treatment approach between the different centres and a limited inclusion in clinical trials. Through better cooperation of the different centres within the BSPHO (Belgian Society of Pediatric Haematology Oncology), important steps are already taken in attracting more clinical studies to our country. We emphasise the need not only to further elaborate this, but also to establish early-phase clinical trials within this collaboration.

\section{Declarations}

Funding - No funds, grants, or other support was received.

\section{Conflicts of interest - none.}

Availability of data - The datasets generated during and/or analysed during the current study are available from the corresponding author on reasonable request.

Authors' contributions - All authors contributed to the study conception and design. Data collection was performed by Dries Ruttens, data analysis was performed by Dries Ruttens and Julie Messiaen. The first 
draft of the manuscript was written by Dries Ruttens and all authors commented on previous versions of the manuscript. All authors read and approved the final manuscript.

Ethics approval - This study was performed in line with the principles of the Declaration of Helsinki.

Approval was granted by the Ethics Committees of the different centres.

Consent to participate - not applicable.

Consent for publication - not applicable.

ACKNOWLEDGEMENTS

We thank Morgan Collin, Caroline De Schepper, Annouschka Laenen, Caroline Martin, An Michiels, Wiert Robberechts, Catherine Sondag, Sam Van Gerwen, Merry Van Puyvelde, Michel Vanden Eynden and Nancy Van Damme for their contribution to this study.

\section{References}

1. Hargrave D, Bartels U, Bouffet E. Diffuse brainstem glioma in children: critical review of clinical trials. The Lancet Oncology. 2006;7(3):241-8.

2. Jansen $M H$, van Vuurden DG, Vandertop WP, Kaspers GJ. Diffuse intrinsic pontine gliomas: a systematic update on clinical trials and biology. Cancer Treat Rev. 2012;38(1):27-35.

3. Veldhuijzen van Zanten SE, Jansen MH, Sanchez Aliaga E, van Vuurden DG, Vandertop WP, Kaspers GJ. A twenty-year review of diagnosing and treating children with diffuse intrinsic pontine glioma in The Netherlands. Expert Rev Anticancer Ther. 2015;15(2):157-64.

4. Green AL, Kieran MW. Pediatric brainstem gliomas: new understanding leads to potential new treatments for two very different tumors. Curr Oncol Rep. 2015;17(3):436.

5. Robison NJ, Kieran MW. Diffuse intrinsic pontine glioma: a reassessment. J Neurooncol. 2014;119(1):7-15.

6. Vitanza NA, Monje M. Diffuse Intrinsic Pontine Glioma: From Diagnosis to Next-Generation Clinical Trials. Curr Treat Options Neurol. 2019;21(8):37.

7. Baugh $\mathrm{J}$ et al. The international diffuse intrinsic pontine glioma registry: an infrastructure to accelerate collaborative research for an orphan disease. J Neurooncol. 2017;132(2):323-31.

8. Puget $\mathrm{S}$ et al. Biopsy in a series of 130 pediatric diffuse intrinsic Pontine gliomas. Child's Nervous System. 2015;31(10):1773-80. 
9. Gupta N, Goumnerova LC, Manley P et al. Prospective feasibility and safety assessment of surgical biopsy for patients with newly diagnosed diffuse intrinsic pontine glioma. Neuro-Oncology. 2018;20(11):1547-55.

10. Paugh BS, Qu C, Jones C et al. Integrated Molecular Genetic Profiling of Pediatric HighGrade Gliomas Reveals Key Differences With the Adult Disease. J Clin Oncol. 2010;28(18):3061-8.

11. Wu G, Broniscer A, McEachron TA et al. Somatic Histone H3 Alterations in Paediatric Diffuse Intrinsic Pontine Gliomas and Non-Brainstem Glioblastomas. Nat Genet. 2012;44(3):251-3.

12. Castel D, Philippe C, Calmon R et al. Histone H3F3A and HIST1H3B K27M mutations define two subgroups of diffuse intrinsic pontine gliomas with different prognosis and phenotypes. Acta Neuropathol. 2015;130(6):815-27.

13. Janssens GO, Jansen MH, Lauwers SJ et al. Hypofractionation vs Conventional Radiation Therapy for Newly Diagnosed Diffuse Intrinsic Pontine Glioma: A Matched-Cohort Analysis. Int J Radiat Oncol Biol Phys. 2013;85(2):315-20.

14. Louis DN, Perry A, Reifenberger G et al. The 2016 World Health Organization Classification of Tumors of the Central Nervous System: a summary. Acta Neuropathol. 2016;131(6):803-820.

15. El-Khouly FE, Veldhuijzen van Zanten SEM, Santa-Maria Lopez V et al. Diagnostics and treatment of diffuse intrinsic pontine glioma: where do we stand? J Neurooncol. 2019;145(1):177-84.

16. Cage TA, Samagh SP, Mueller S et al. Feasibility, safety, and indications for surgical biopsy of intrinsic brainstem tumors in children. Child's Nervous System. 2013;29(8):1313-9.

17. Gallitto M, Lazarev S, Wasserman I et al. Role of Radiation Therapy in the Management of Diffuse Intrinsic Pontine Glioma: A Systematic Review. Adv Radiat Oncol. 2019;30(4):520-31.

18. Freese C, Takiar V, Fouladi M, DeWire M, Breneman J, Pater L. Radiation and subsequent reirradiation outcomes in the treatment of diffuse intrinsic pontine glioma and a systematic review of the reirradiation literature. Pract Radiat Oncol. 2017;7(2):86-92.

19. Janssens GO, Gandola L, Bolle S et al. Survival benefit for patients with diffuse intrinsic pontine glioma (DIPG) undergoing re-irradiation at first progression: A matched-cohort analysis on behalf of the SIOP-E-HGG/DIPG working group. Eur J Cancer. 2017;73:38-47.

20. Mathew RK, Rutka JT. Diffuse Intrinsic Pontine Glioma : Clinical Features, Molecular Genetics, and Novel Targeted Therapeutics. Journal of Korean Neurosurgical Society. 2018;61(3):343-51.

21. Lu VM, Power EA, Zhang L, Daniels DJ. Liquid biopsy for diffuse intrinsic pontine glioma: an update. J Neurosurg Pediatr. 2019;24(5):593-600. 
22. Huang TY, Piunti A, Lulla RR et al. Detection of Histone H3 Mutations in Cerebrospinal Fluid-Derived Tumor DNA From Children With Diffuse Midline Glioma. Acta neuropathologica communications. 2017;5(1):28.

23. Broniscer A, Laningham FH, Sanders RP, Kun LE, Ellison DW, Gajjar A. Young age may predict a better outcome for children with diffuse pontine glioma. Cancer. 2008;113(3):566-72.

24. Cohen ME, Duffner PK, Heffner RR, Lacey DJ, Brecher M. Prognostic factors in brainstem gliomas. Neurology. 1986;36(5):602-5.

\section{Tables}

Due to technical limitations, table 1, 2 is only available as a download in the Supplemental Files section.

\section{Figures}

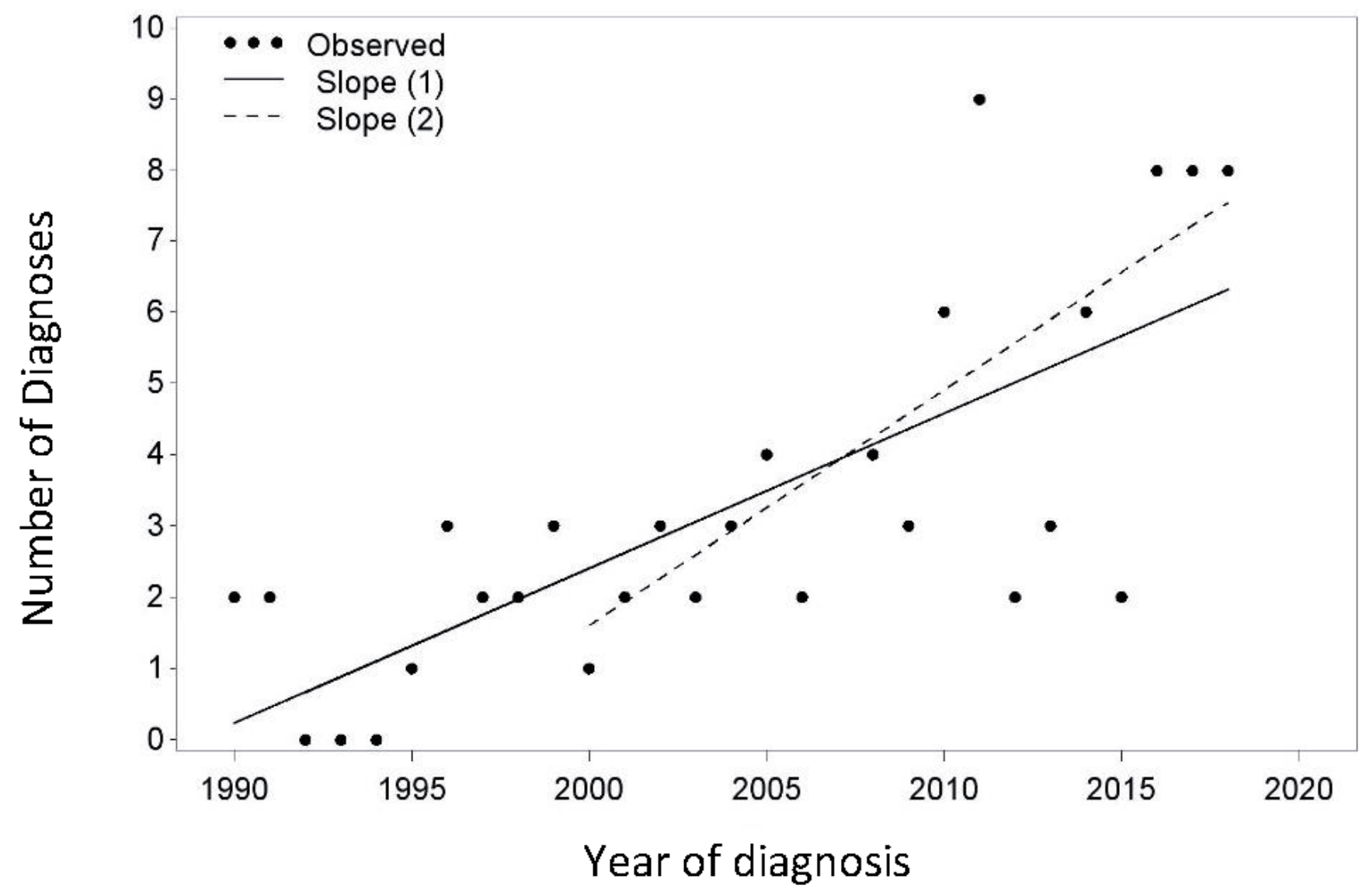

\section{Figure 1}

New diagnosed cases per year (dots) and estimated slopes over time. Slope 1: all years included, patients from one centre excluded. Slope 2: patients from all centres included from 2000 onwards. 


\section{Figure 2a}

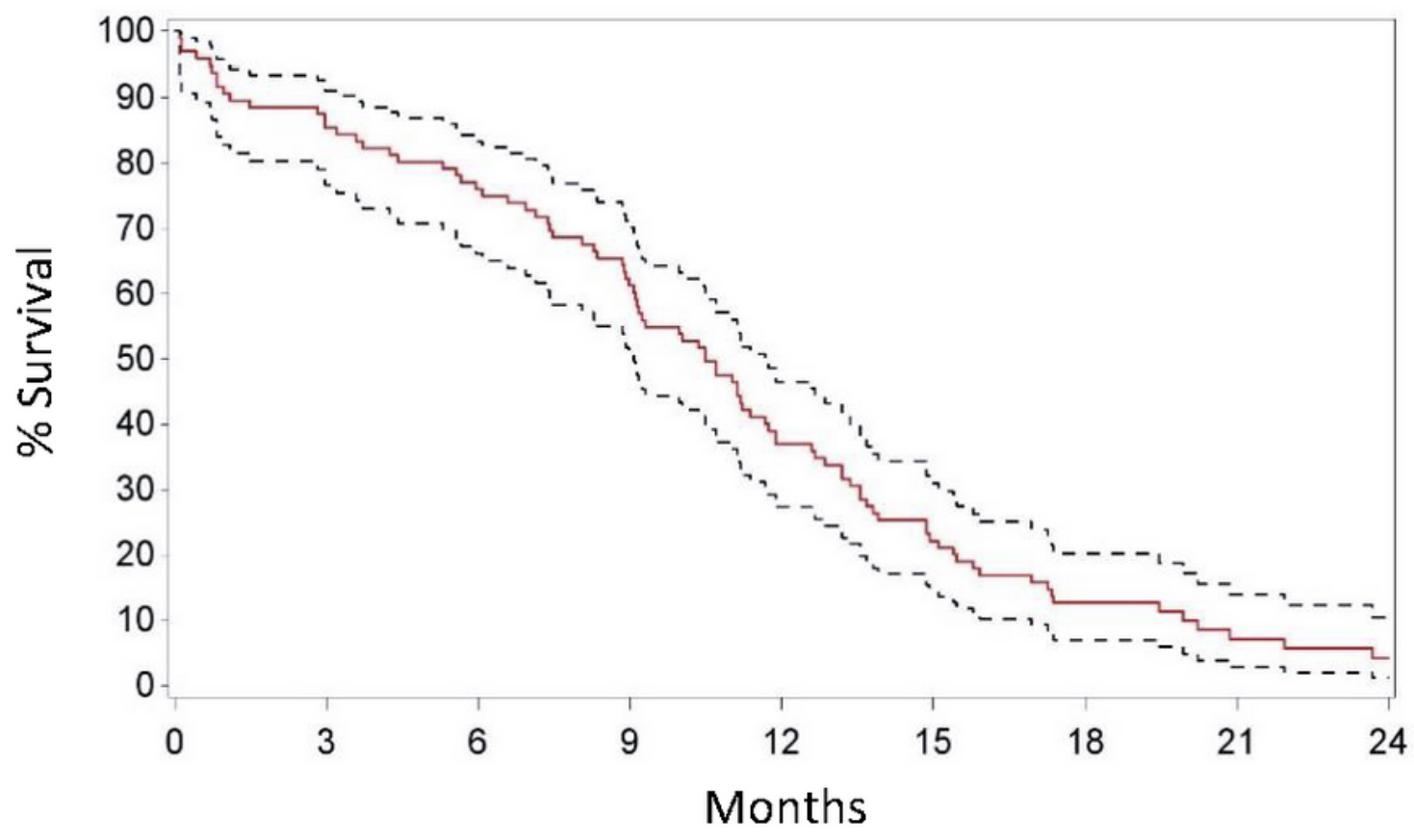

Figure 2b

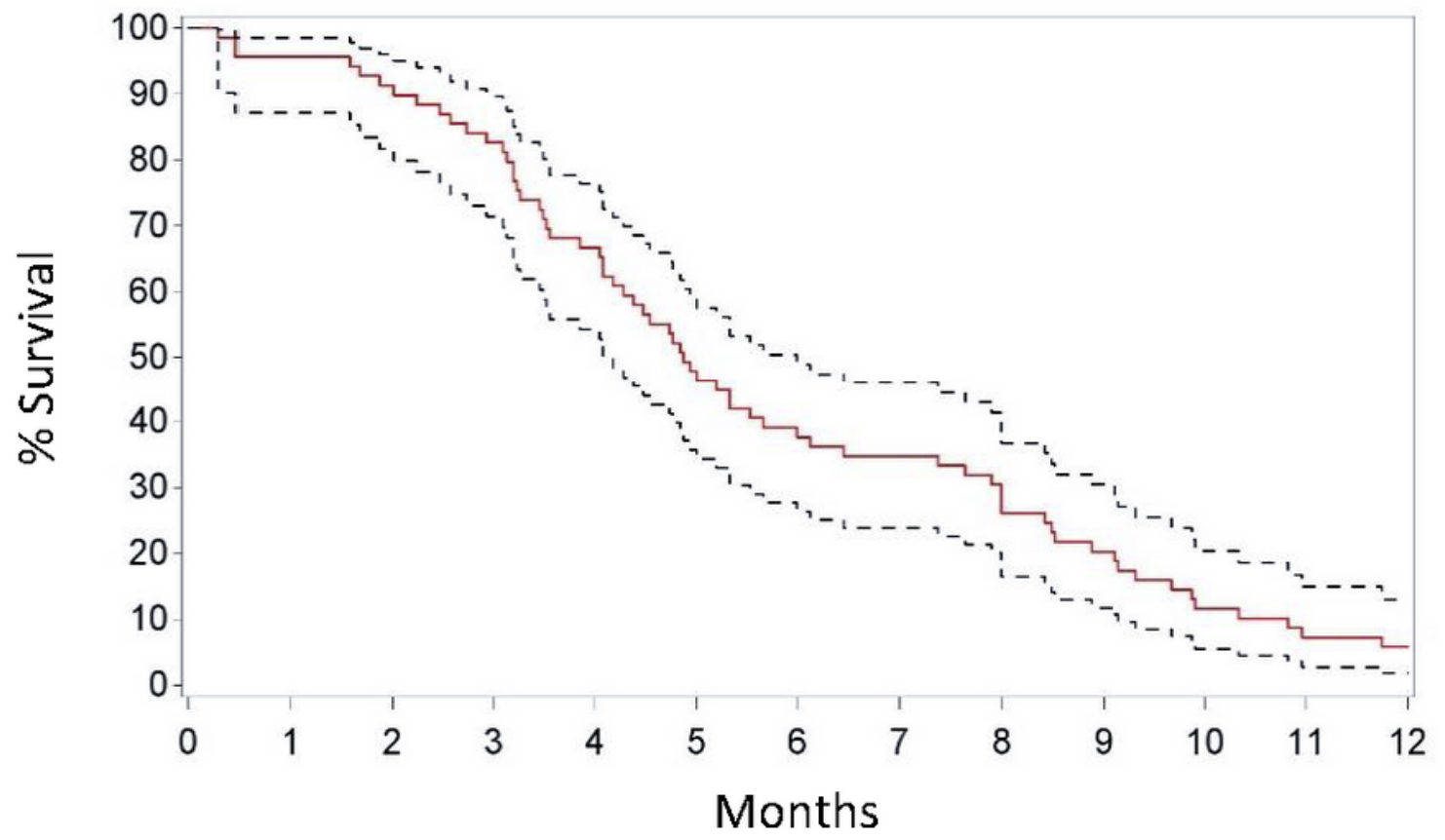

Figure 2

a) Kaplan-Meier plot of the estimated overall survival rate of study patients. b) Kaplan-Meier plot of progression-free survival rate of study patients 


\section{Figure 3a}

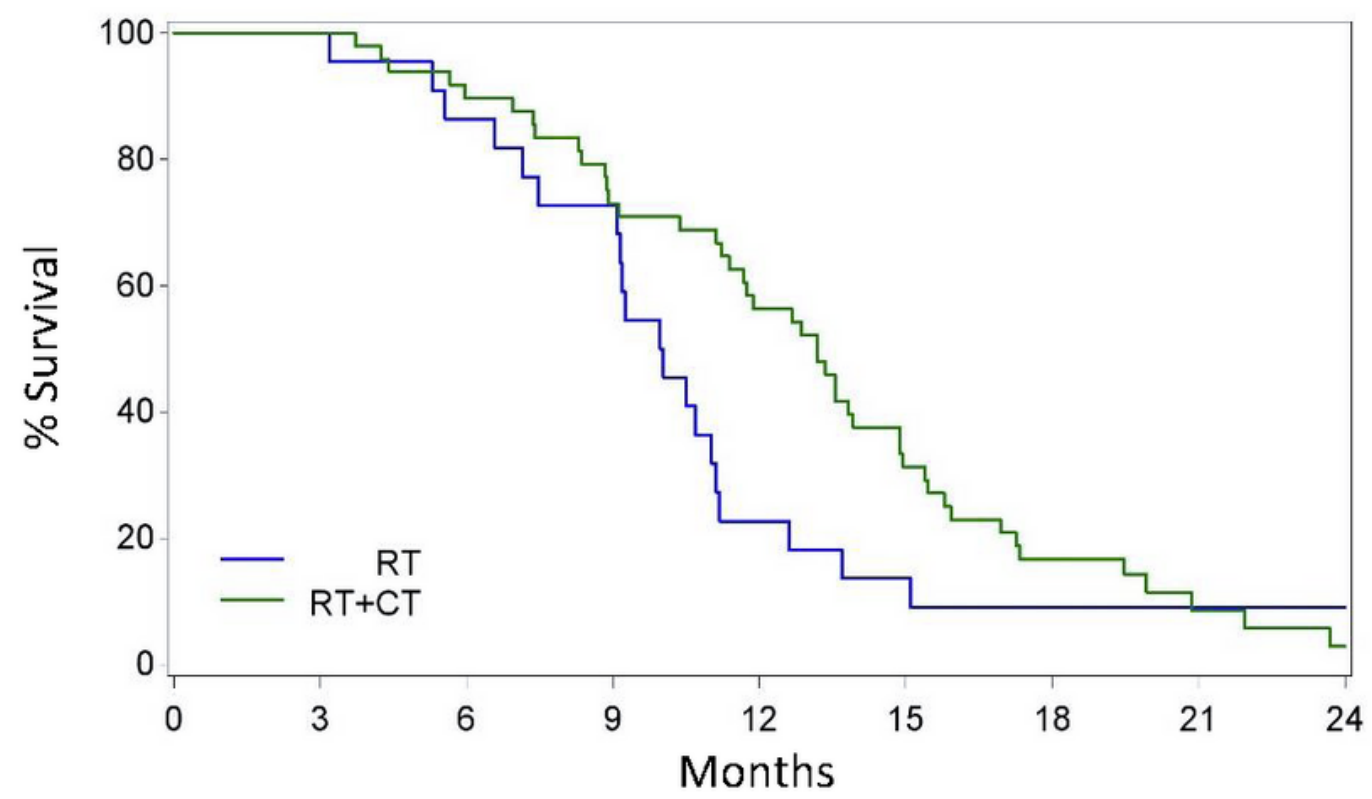

Figure 3b

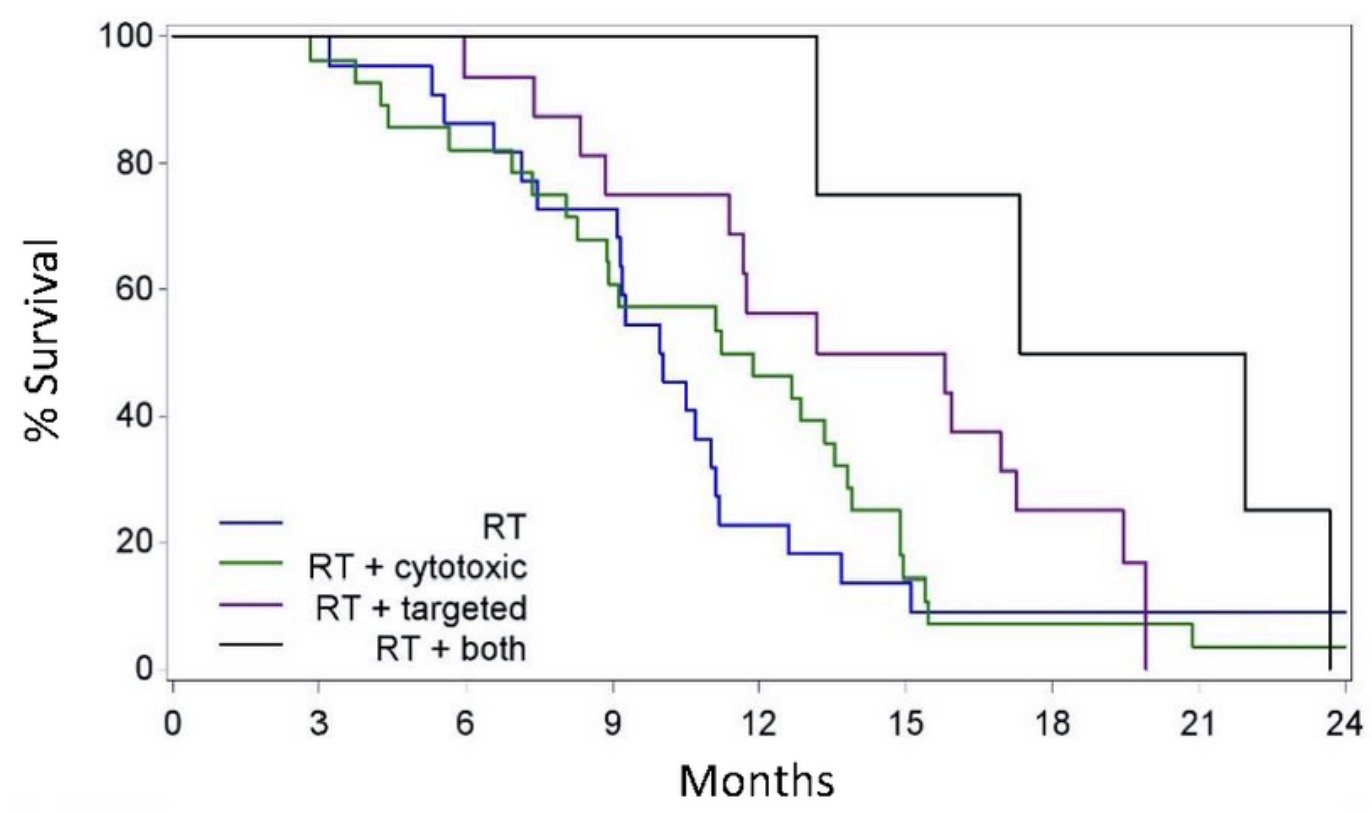

Figure 3

a) OS rates of patients treated with RT alone (blue curve) vs RT and chemotherapy (green curve). b) OS rates of patients treated with RT alone (blue curve) or with a combination of RT and cytotoxic therapy (green curve) or targeted therapy (purple curve) or both (black curve)

\section{Supplementary Files}


This is a list of supplementary files associated with this preprint. Click to download.

- Table1.pdf

- Table2.pdf 